\title{
Ultra-high-Resolution, Label-Free Hyperlens Imaging in the Mid-IR
}

\section{Mingze He}

Vanderbilt University https://orcid.org/0000-0001-8773-1268

\section{Ganjigunte lyer}

U. S. Naval Research Laboratory

\section{Shaurya Aarav}

Princeton University

\section{Sai Sunku}

Columbia University

\section{Alexander Giles}

US Naval Research Laboratory

Thomas Folland

The University of lowa

\section{Nicholas Sharac}

U. S. Naval Research Laboratory

\section{Xiaohang Sun}

Princeton University

Joseph Matson

Vanderbilt University https://orcid.org/0000-0002-7183-2686

\section{Song Liu}

Kansas State University https://orcid.org/0000-0002-3046-3335

\section{James Edgar}

Kansas State University https://orcid.org/0000-0003-0918-5964

\section{Jason Fleischer}

Princeton University https://orcid.org/0000-0003-2453-2581

\section{Dimitri Basov}

Columbia University

Joshua Caldwell ( $\nabla$ josh.caldwell@vanderbilt.edu )

Vanderbilt University, Mechanical Engineering Department https://orcid.org/0000-0003-0374-2168

\section{Article}

Keywords: hyperlens imaging, image reconstruction methodologies 
Posted Date: March 3rd, 2021

DOl: https://doi.org/10.21203/rs.3.rs-264584/v1

License: (c) (i) This work is licensed under a Creative Commons Attribution 4.0 International License. Read Full License 
Ultra-high-Resolution, Label-Free Hyperlens Imaging in the Mid-IR

Mingze $\mathrm{He}^{1 \dagger}$, Ganjigunte R. S. Iyer ${ }^{2,3 \dagger}$, Shaurya Aarav ${ }^{4 \dagger}$, Sai. S. Sunku ${ }^{5}$, Alexander. J. Giles ${ }^{3}$, Thomas. G. Folland $^{1,6}$, Nicholas Sharac ${ }^{2}$, Xiaohang Sun ${ }^{4}$, Joseph Matson ${ }^{1}$, Song Liu ${ }^{7}$, James H. Edgar ${ }^{7}$, Jason W. Fleischer $^{4}$, D.N. Basov ${ }^{5}$ and Joshua D. Caldwell ${ }^{1 *}$

1. Mechanical Engineering Department, Vanderbilt University, Nashville, TN 37212, USA.

2. ASEE/NRC Postdoctoral Fellow residing at NRL, Washington DC 20375, USA.

3. U. S. Naval Research Laboratory, Washington DC 20375, USA.

4. Department of Electrical Engineering, Princeton University, NJ, 08544, USA.

5. Department of Physics, Columbia University, New York, New York 10027, USA.

6. Department of Physics and Astronomy, The University of Iowa, Iowa City, Iowa, 52242 USA.

7. Tim Taylor Department of Chemical Engineering, Kansas State University, Manhattan, Kansas 66506, USA.

$\dagger$ denotes equal contributions

* email: josh.caldwell@ vanderbilt.edu

\section{Abstract}

The hyperbolic phonon polaritons supported in hexagonal boron nitride (hBN) exhibiting long scattering lifetimes are advantageous for applications like super-resolution imaging via hyperlensing. Yet, challenges exist in hyperlens imaging to distinguish individual and closely spaced objects and in correlating the complicated hyperlens fields with the structure of an unknown object underneath the hyperbolic material. Here, we make significant strides to overcome each of these three challenges. For the first two, we demonstrate that monoisotopic $h^{11} \mathrm{BN}\left(>99 \%{ }^{11} \mathrm{~B}\right)$ provides the ability to experimentally resolve structures as small as 40-nm and those with sub-25-nm spacings, inferring at least 154- and 270- times smaller than free-space wavelength, showing improvements in spatial resolution. We also present an image reconstruction algorithm that provides a structurally accurate, visual representation of the embedded objects using only the hyperbolic dielectric function and thickness as input parameters. Further, we offer additional insights into the frequency dependence for realizing optimal hyperlens performance. Thus, our results significantly advance label-free, highresolution, spectrally selective hyperlens imaging and image reconstruction methodologies. 


\section{Introduction}

Sub-diffractional imaging in conventional optical microscopy is not possible due to Abbe's diffraction limit, as light scattered from deeply sub-wavelength objects rapidly decay from the surface, resulting in evanescent fields that do not propagate into the far-field. The hyperlens concept provides a promising approach to overcome this challenge. This approach exploits hyperbolic polaritons (HPs) supported in highly anisotropic materials exhibiting a dielectric permittivity tensor that is opposite in sign along orthogonal directions, e.g., $\left(\varepsilon_{t}>0, \varepsilon_{z}<0\right)$ or $\left(\varepsilon_{t}<0, \varepsilon_{z}>0\right)$, where $\varepsilon_{t}=\varepsilon_{x}=\varepsilon_{y}$ and $\varepsilon_{z}$ are tangential and axial permittivities, respectively ${ }^{1-3}$. In the anisotropic region, high- $k$ wavevectors can propagate at an angle consistent with the material dispersion, and the angle $\theta$ can be defined to the surface normal (z-axis) ${ }^{4-}$ $6:$

$$
\theta=\pi / 2-\arctan \left(\sqrt{\varepsilon_{z}} / i \sqrt{\varepsilon_{t}}\right)
$$

The scattering off of a sub-diffractional object can therefore launch HPs into the hyperbolic slab, which will then propagate at this frequency-dependent angle to the opposite surface where they can be directly probed (Fig. 1a). For a curved hyperbolic material ${ }^{7,8}$, these hyperbolic "rays" propagating along the radial directions are expanded, resulting in magnified hyperlens fields that can become resolvable in the far-field when the features expand beyond the diffraction limit. This is also possible through (flat) metalens designs $^{9,10}$, or in the near-field via probes such as scattering-type scanning near-field optical microscopy (s$\mathrm{SNOM})^{5,6}$, which we have employed here.

Hyperlensing for sub-diffraction-limited imaging was originally demonstrated with artificial hyperbolic metamaterials (HMMs) ${ }^{7}$, i.e., metal and dielectric stacks ${ }^{7,8}$. However, these HMMs suffer from high optical losses inherent in the metallic layers, which limits the transmission efficiency and the maximum wavevectors accessed, restricting the minimum resolvable feature size. In contrast, natural hyperbolic materials $5,6,11,12$, such as hexagonal boron nitride $(\mathrm{hBN})$, have considerablly reduced optical losses. The 
hyperbolicity of hBN comes from two sets of optic phonons that are spectrally separated, giving rise to two distinct Reststrahlen bands (upper, URB and lower, LRB) where $\operatorname{Re}(\varepsilon)<0$ along at least one axis. Within these bands hyperbolic phonon polaritons (HPhPs) are supported, enabling hyperlens imaging ${ }^{5,6,11}$. Although hBN exhibits reduced optical loss compared with $\mathrm{HMMs}^{5,6,11}$, the spatial resolution and the $\mathrm{HPhPs}$ transmission are still limited. Further, instead of magnified images, the hyperlens fields collected represent complex products of (sub-wavelength) diffraction, posing challenges to identify the imaged objects without prior knowledge.

Here we address these challenges by combining material developments with an image reconstruction algorithm. With the implementation of monoisotopic $\mathrm{h}^{11} \mathrm{BN}(>99 \%)$ we reduce the optical loss by threefold, resolving objects as small as 44-nmm in diameter and interparticle gaps of $<25-\mathrm{nm}$. Based on the smallest object imaged, we report a normalized resolution of at least $\lambda_{F S} / 154$, where $\lambda_{F S}$ is the free-space wavelength of the incident light, representing a nearly four-fold improvement compared with previous reports of hyperlens imaging using naturally abundant (NA) $\mathrm{hBN}^{5,6}$. Equally promising, the hyperlens fields from monoisotopic $\mathrm{hBN}$ are significantly improved due to the higher transmission efficiency, tripling the peak-signal-to-noise ratio (PSNR) over NA hBN for slab of the same thickness. Moreover, we also provide and demonstrate a backpropagation method to identify spatial locations and sizes of unknown objects from the hyperlens fields, using only the dielectric function and thickness of $\mathrm{hBN}$ as input parameters enabling the potential for on-the-fly conversion of the collected hyperlens fields into object images. In addition, we also offer insights into the frequency dependence for optimizing hyperlens performance. The combination of monoisotopic $\mathrm{h}^{11} \mathrm{BN}$ and computational image processing highlighted here therefore provides significant advancements towards practical mid-infrared (MIR) hyperlens designs, and lays the foundation for future works investigating the potential for practical spectroscopic hyperlens imaging.

\section{RESULTS}

In the simplest case, the frequency-dependent angular propagation of the HPhPs can be visualized using a ray-tracing picture (Fig. 1a). Here, evanescent fields are produced as long-wavelength MIR light is 
scattered by a sub-diffractional object, which launches propagating HPhPs due to the requisite momentum and the proximity to the hyperbolic slab. These volume-confined HPhPs propagate at the frequency-dictated angle through the slab (Eq. 1) until they reach the opposite surface ${ }^{1}$, where they are reflected (Fig. 1a). Although sub-wavelength modes cannot propagate into free space, this reflection at the top surface results in evanescent fields that extend just above the surface of the hyperbolic slab that can be probed via external means sensitive to near-fields, such as s-SNOM ${ }^{5,6}$. For frequencies close to that of the longitudinal optic (LO) phonon of hBN (the upper bound of the hBN URB), the propagation angle will be near normal (Fig. 1e), resulting in hyperlens fields probed on the hBN top surface that approximates a slightly magnified replica of the underlying object. On the other hand, reducing the illumination frequency increases the propagation angles of the launched $\mathrm{HPhP}$ rays, leading to an enlarged hot-ring diameter $\mathrm{D}(\omega)$ and a wider separation between concentric rings, as shown in Fig. 1b-d.

To demonstrate this experimentally, we fabricated a series of nickel structures by electron beam lithography and a standard lift-off procedure (see methods), afterwhich flakes of NA (125-nm thick) and ${ }^{11}$ B-enriched (>99\%) hBN (125 nm thick) were mechanically exfoliated and transferred on top of these nickel disks. While both ${ }^{10} \mathrm{~B}$ - and ${ }^{11} \mathrm{~B}$-enriched $\mathrm{hBN}$ have been grown with high purity ${ }^{13-15}$ with enhanced phonon lifetimes and propagation lengths over NA materials ${ }^{16,17}, \mathrm{~h}^{11} \mathrm{BN}\left(>99 \%{ }^{11} \mathrm{~B}\right)$ was chosen for these studies due to the near-negligible spectral shift in the optic phonons, i.e., $\left(\omega_{T O} \sim 1359.8, \omega_{L O} \sim 1608.7 \mathrm{~cm}^{-1}\right)$ with respect to NA material $\left(\omega_{T O} \sim 1360, \omega_{L O} \sim 1614 \mathrm{~cm}^{-1}\right)$. Therefore, this small variation in the spectral response allows direct comparison between the NA and isotopically enriched hyperlenses at the same incident frequencies while preserving roughly equal values for the real part of the permittivity. Here, we have chosen $125 \mathrm{~nm}$ flakes for both the $\mathrm{h}^{11} \mathrm{BN}$ and NA hBN slabs, therefore isolating the role that loss reduction plays in improving hyperlens performance. 


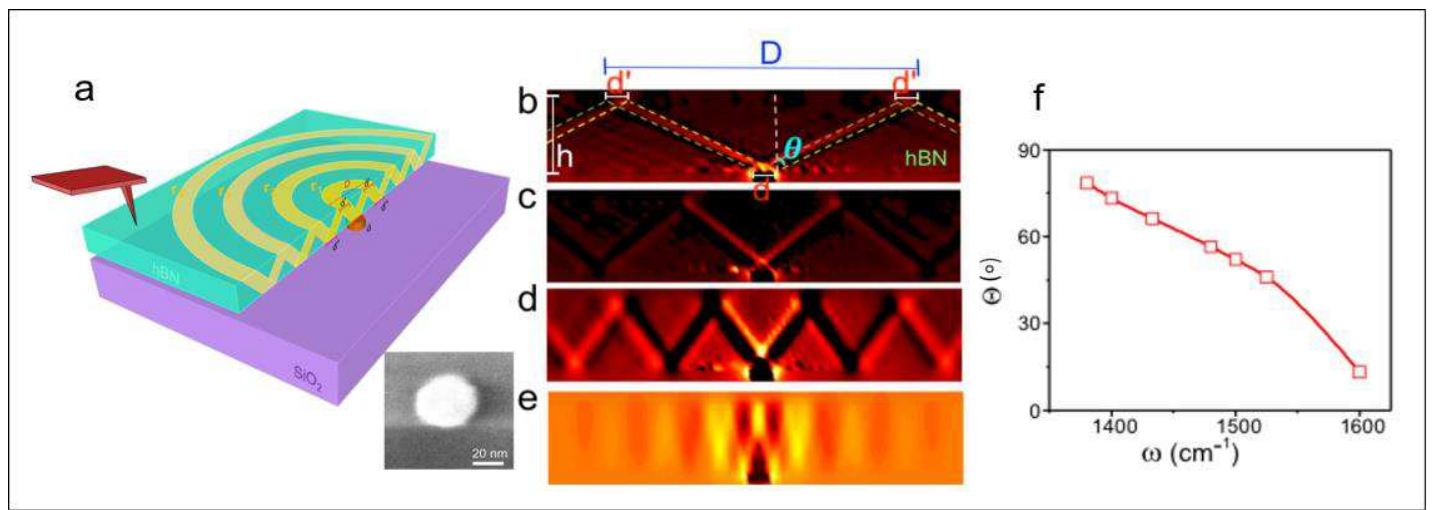

Fig. 1: Sub-diffractional focusing and imaging through hBN a Schematic illustration of the hot rings/fringes on the top surface and within the $h B N$ as a result of multiple reflection of the HPhPs launched from the edges of the sub-diffractional nickel disks embedded beneath 125-nm thick $h^{11} B N$ slab. Inset: a SEM image of the 44-nm disk. b-e The simulated cross-sectional E-field distribution for a 40-nm disk embedded under a 125-nm thick $h^{11} B N$ flake at $\omega=1433 \mathrm{~cm}^{-1}, 1500 \mathrm{~cm}^{-1}, 1560 \mathrm{~cm}^{-1}, 1600 \mathrm{~cm}^{-1}$ respectively. $f$, The calculated propagation angle $(\theta)$ of HPhPs within $h^{11} B N$ as a function of frequency. Open squares correspond to spectral positions for experiments and simulations discussed.

\section{Hyperlens fields of isolated and closely spaced structures}

To compare the imaging performance of $\mathrm{NA} \mathrm{hBN}$ and $\mathrm{h}^{11} \mathrm{BN}$, we first measured the hyperlens fields resulting from scattering of a MIR light by a 44-nm diameter disk embedded beneath each of the two hBN slabs, collected via s-SNOM (Fig. 2a-b). The lower losses of $\mathrm{h}^{11} \mathrm{BN}$ (Fig. 2a) lead to a stronger s-SNOM amplitude contrast than NA hBN at the same frequencies (Fig. 2b). Further, the longer propagation lengths of the $\mathrm{h}^{11} \mathrm{BN} \mathrm{HPhPs}$ increase in the number of internal reflections (and thus, more concentric hot-rings) that occur before the HPhPs decay (Fig. 2a,c vs. Fig. 2b,d). To quantitatively compare the transmission efficiency between the two forms of hBN, we benchmark the peak signal-to-noise ratio (PSNR) of the two hyperlens systems. While the noise levels for both s-SNOM images are very similar (details included in SI, section IV; 0.011 for NA hBN versus 0.014 for $h^{11} \mathrm{BN}$ ), the amplitude of the collected signal from the $\mathrm{h}^{11} \mathrm{BN}$ hyperlens is much stronger. Specifically, line scans from this hyperlens field (Fig. S4a) indicate that the signal is far above the noise level, resulting in a PSNR of 11.5. In contrast, the comparable hyperlens fields using NA hBN are just resolvable due to low signal strength, with a corresponding PSNR of only

3.64 (Fig. S3, Fig. S4a, Table S1). As such, the ability to resolve such small structures at $\omega=1480 \mathrm{~cm}^{-1}\left(\lambda_{\mathrm{FS}}\right.$ 

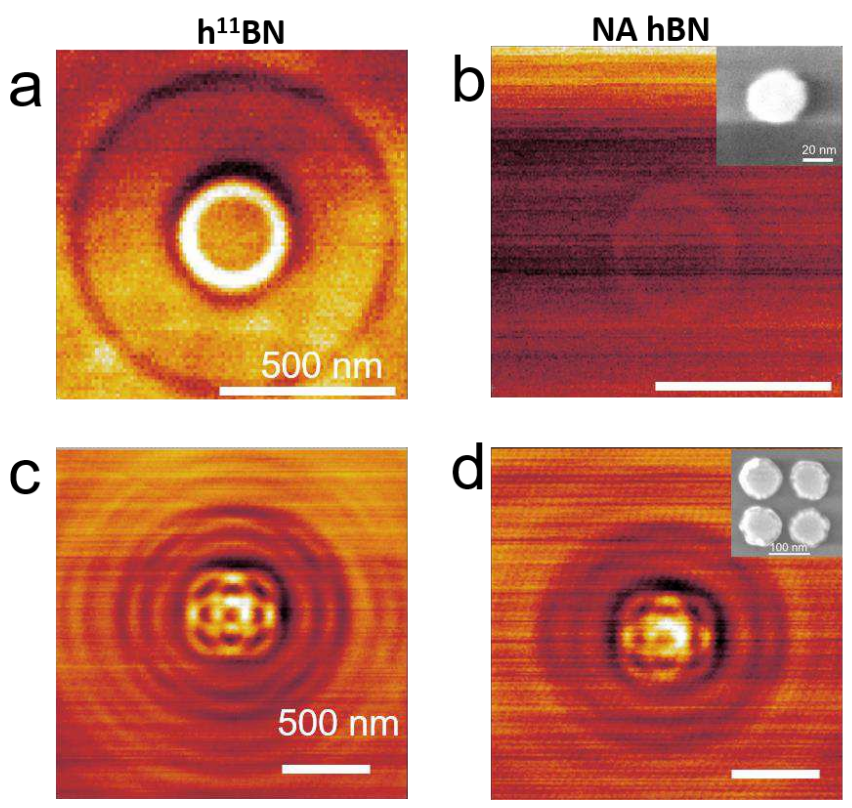

Fig. 2: Experimental demonstration of super high-resolution sub-diffractional focusing and imaging through natural and monoisotopically enriched $\mathbf{h B N}$. (a,b). Near field amplitude image of $44 \mathrm{~nm}$ square at $\omega=1500 \mathrm{~cm}^{-1}$ for ${ }^{11} B$ and NA-hBN. (c, d) Near field amplitude image of a $2 \times 2,100 \mathrm{~nm}$ disk array with $25 \mathrm{~nm}$ gap separation at $\omega=1480 \mathrm{~cm}^{-1}$ for $^{11} B$ and NA-hBN. Corresponding SEM images are inseted.
$=6.76 \mu \mathrm{m})$ implies sub-

diffractional imaging resolution of at least $\lambda_{\mathrm{FS}} / 154$ for both hyperlens systems, a nearly four-fold improvement over previous reports with NA $\mathrm{hBN}^{5,6}$. Based on the comparative PSNR values, the $\lambda_{\mathrm{FS}} / 154$ do not represent the imaging power limitations of the monoisotopic hyperlens, but instead represent simply the results from the smallest

structures realized in this study. Thus, it is anticipated that the $\mathrm{h}^{11} \mathrm{BN}$ hyperlens can provide substantial additional resolving power for even smaller objects, while the NA device is at or close to its resolving power. This overall improvement via $\mathrm{h}^{11} \mathrm{BN}$ thereby facilitates the transition to thicker and/or curved films that could enable sufficient magnification for far-field hyperlens approaches.

For practical use, a hyperlens must resolve not only isolated objects, but also multiple closely spaced structures. To validate this capability, we consider a $2 \times 2$ array of 100-nm diameter, 30-nm tall nickel disks with nominal separation distances ranging from 25 to $125 \mathrm{~nm}$ (a top-view SEM image of the smallest gap array is shown in the inset of Fig. 2d). The corresponding hyperlens fields were collected via s-SNOM for both the $\mathrm{h}^{11} \mathrm{BN}$ and NA-hBN flakes with an incident laser at $\omega=1480 \mathrm{~cm}^{-1}$, as provided in Fig. $\mathbf{2 c}$ and $\mathbf{2 d}$, respectively. Consistent with the imaging of single disks, the image quality is superior for those collected through the $\mathrm{h}^{11} \mathrm{BN}$ slab. Furthermore, line scans across the disks, collected along the center and diagonal 
directions, reveal an even higher PSNR (37.75) for the $\mathrm{h}^{11} \mathrm{BN}$ hyperlens compared to the NA slab (11.32, see Fig. S4b and Table S1).

\section{Image reconstruction algorithm}

While these efforts highlight improvements in the PSNR for the collected hyperlens fields, directly identifying the structure of underlying objects from these measurements is still challenging. Because $\mathrm{HPhP}$ rays are launched from all edges of the object into all directions, the resultant concentric patterns give rise to complicated fields even for simple systems such as an isolated disk (Fig. 2a). For more complex objects or multiple closely spaced structures, such patterns highlight extensive interference between the overlapping HPhP rays, making the collected fields complicated and not easily identifiable as the objects they represent (Fig. 2c). In other words, the measured fields are not direct images and thus must be reconstructed into an image of the underlying object for the hyperlens concept to be useful.

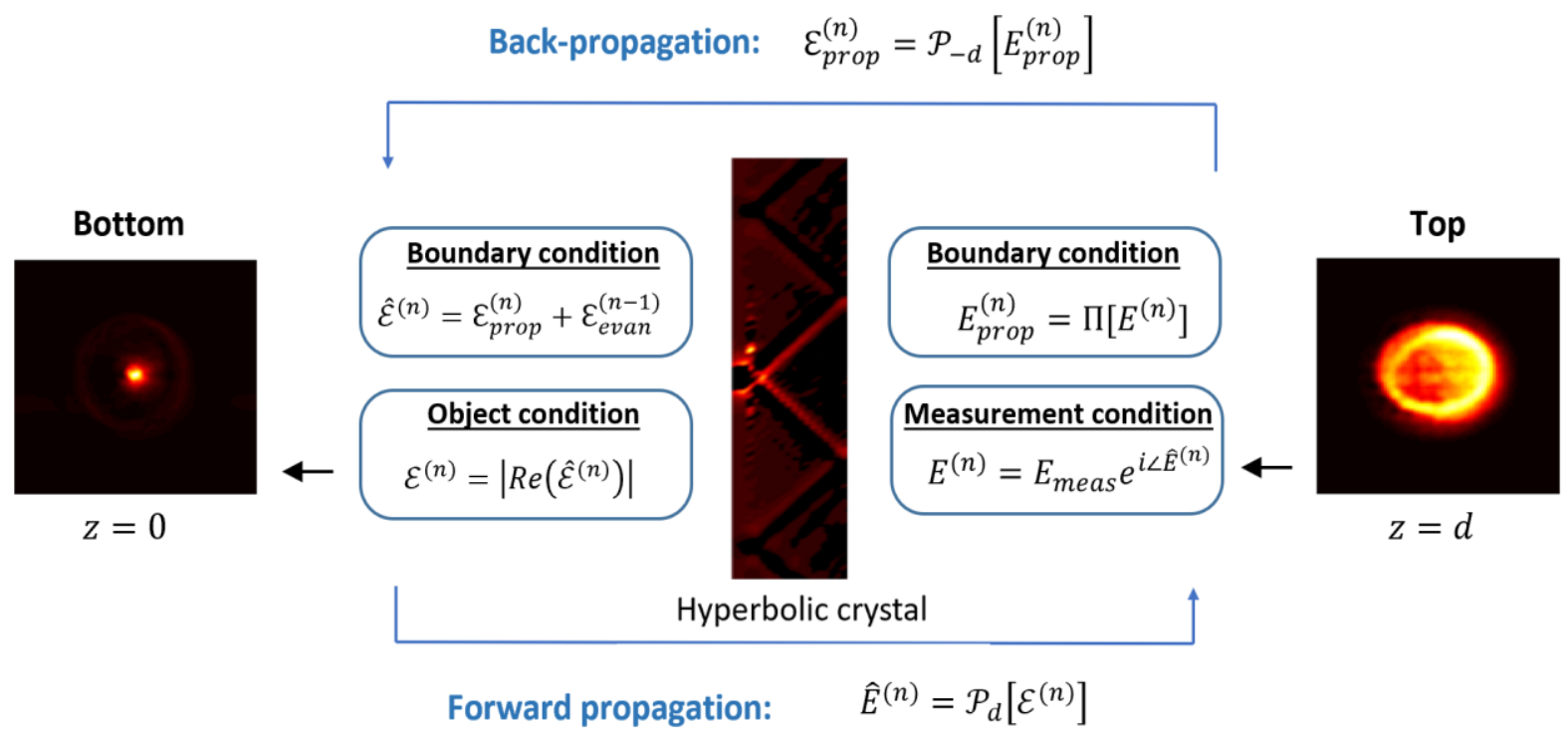

Fig. 3: Algorithm flowchart. The algorithm iterates between the experimentally measured field amplitude at the top of the crystal and the object field at the bottom of the crystal. The right-most image shows a sample measurement of the crystal output, the central image depicts approximate ray paths within the crystal, and the leftmost image represents the input field reconstruction after 200 iterations. We start the algorithm by enforcing the measurement condition; $E_{\text {meas }}$ is the measured field, $\angle$ denotes the phase of the complex field (to be found), and $n$ represents the iteration number. The projection operator $\Pi$ extracts the propagating modes from $E^{(n)}$ by enforcing the boundary constraints on the input and output faces of the crystal. The angularspectrum propagation operator $\mathcal{P}_{d}$ transports the propagating modes of the input field by a distance $d$. To match the boundary conditions at the bottom of the crystal, we add the evanescent modes (obtained from the previous iteration $\hat{\mathcal{E}}^{(n-1)}$ ) to get the total field $\hat{\mathcal{E}}^{(n)}$. We further apply the small object constraint to get our reconstruction. Subscripts prop and evan represent 
propagating and evanescent components of a given electric field, respectively, $\mathcal{R} e(\cdot)$ represents the real part of $(\cdot)$, and || represents an absolute value operation.

To address this challenge, we use the angular spectrum method to back-propagate the measured fields at the top surface of the hBN slab to the unknown scattering source at the bottom surface. The algorithm is a modified Gerchberg-Saxton (GS) algorithm ${ }^{18}$, as illustrated in the flowchart provided in Fig. 3. In the case of s-SNOM, only the z-component of the E-field, $E_{z}$, is measured by the s-SNOM tip. Nevertheless, propagation in the $\mathrm{hBN}$ is anisotropic, so the full vector near-field of the object must be retrieved. The algorithm starts with the measured s-SNOM image as the top-field profile, and the measured field is back-propagated to the bottom side of $\mathrm{hBN}$ by an inversed propagation function $\mathcal{P}_{-d}$. From there, the field of the bottom object, representing the position and size of the object under hBN, can be extracted. The bottom field is then forward-propagated to the top surface, then again back-propagated to the bottom, and difference between "calculated top field" and "measured top field" is decreased during such an iteration. Iterations are continued until the calculated top field converges (within a pre-determined error) to the measured field distribution from s-SNOM. To match the boundary conditions at the bottom surface, this reconstruction includes evanescent as well as propagating modes. Empirically, we found that the incident field could be approximated with linearly independent plane waves (valid since the object is deeply subwavelength), while the field inside the crystal could be modeled as a joint (scalar) field hybridizing the two polarizations. Forward propagation consists of the following steps: 1) coupling the light from the illumination source into the crystal; 2) advancing the propagating modes to the top surface (an approximation that gets better with increasing slab thickness); and 3) replacing the z-component of the top field with that of the experimentally measured one. More details are given in the SI, section VI.

To test the reconstruction algorithm, hyperlens fields were collected from the $\mathrm{h}^{11} \mathrm{BN}$ hyperlens over the 2x2, 100-nm diameter dot array with the smallest gap $(\sim 25 \mathrm{~nm})$ at various frequencies (Fig. 4a-d). The acquired hyperlens fields are significantly different at each of these frequencies and illustrate consistency with predicted fields via Finite Element Method (FEM) simulations, as shown in Fig. 4e-h. Note that additional speckling is observed in FEM simulations, which is the result of high-order HPhPs superimposed 
upon the fundamental HPhPs that are at the heart of our discussions here. To minimize the speckling so that the simulated hyperlens field is easier to read, the simulations in Fig. $\mathbf{4}$ are performed with artificially increased loss of $\mathrm{h}^{11} \mathrm{BN}$ by three-fold, and FEM simulations with original loss are included in Fig. S9. Although we focus on the s-SNOM amplitude fields in this study, we note that similar behaviors are also shown in s-SNOM phase maps (Fig. S5).

Despite the complexity in the hyperlens fields from the 2x2 disk arrays (Fig. 4), the reconstruction algorithm successfully retrieves images of the underlying structures featuring accurate measurements of the structure size and alignment, as shown in Fig. 4i-l. The extracted diameter and interparticle gaps of the disks, averaged over the four incident frequencies, are $87 \mathrm{~nm}$ and $27 \mathrm{~nm}$, respectively, which are in good agreement with the actual diameter $(\sim 100 \mathrm{~nm})$ and gap values $(\sim 25 \mathrm{~nm})$. While outside of the scope of this

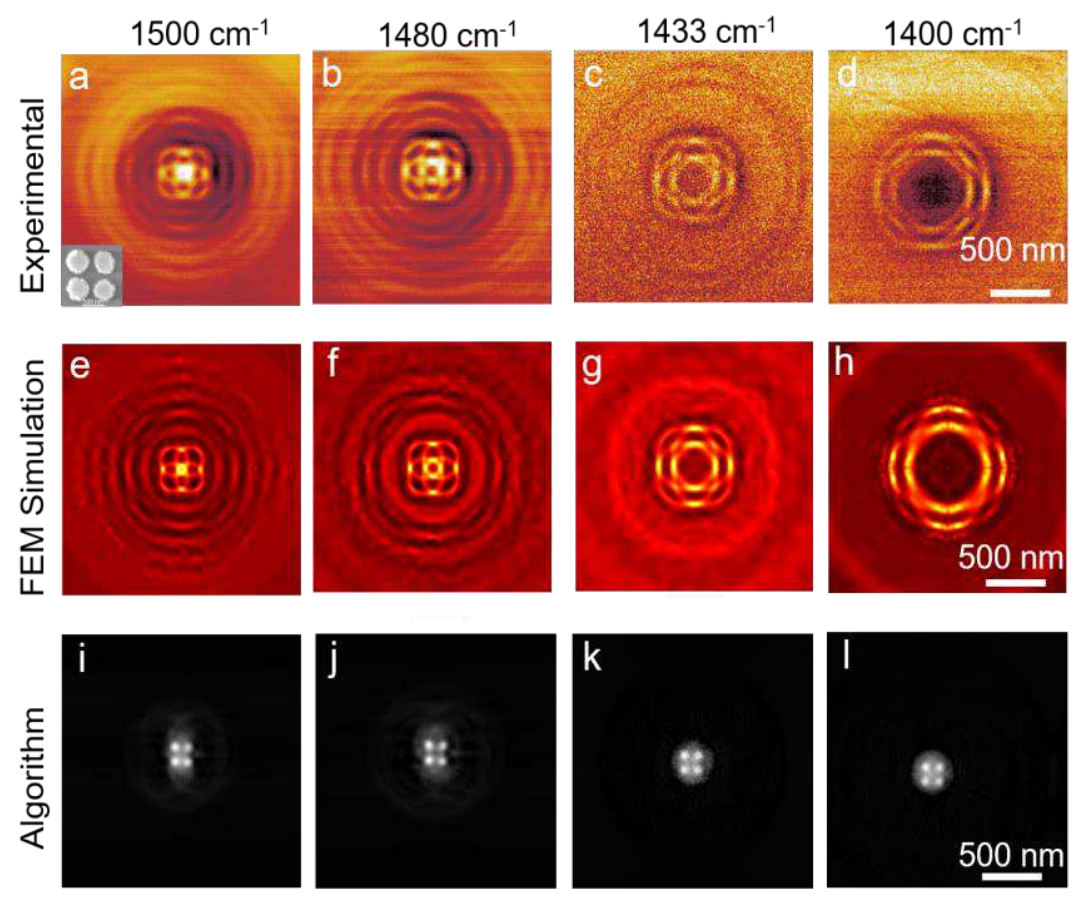

Figure 4: Frequency-dependent hyperlens fields and reconstruction. (a-d) Near-field amplitude images of $100-n m$ disk array with 25-nm inter-particle gaps, showing a strong dependence on the HPhP propagation angle. (e-h) Corresponding FEM simulations. (i-l) Reconstructed underlying structures from the corresponding s-SNOM images.

work, by correlating the multi-frequency measurements within the reconstruction protocol (rather than treating each frequency as an independent measurement), this method can be refined further to give an improved estimate of the underlying structures. The data summarized in Table $\mathbf{S 2}$ can also be used to 
extract other information as well, for instance as a means to fine-tune the material dielectric properties, ${ }^{19}$ such as the recent use of near-field measurements for optimizing the dielectric function of the biaxial hyperbolic cystal $\mathrm{MoO}_{3}{ }^{20}$. Note that we perform this at different frequencies, i.e., different propagation angles of HPhPs (Eq. 1), which is also of significant importance for resonant materials, e.g., biomaterials, since imaging on/off resonance could provide strong material contrast, potentially useful in object material identification as well ${ }^{21,22}$.

\section{Hyperlens imaging figures of merit}

While our image reconstruction algorithm successfully retrieved the underlying image of the embedded objects, realizing far-field IR hyperlenses also requires high image resolution and HPhP transmission. By implementing monoisotopic $\mathrm{h}^{11} \mathrm{BN}$, we have significantly improved the image quality, which also shows a strong dependence on the incident frequency, as shown in Fig. 4. Thus, understanding how those factors influence the hyperlens field quality is imperative for future applications. As the fields collected by sSNOM are derived from HPhPs scattered from the metal/air edge underneath that propagate through the hBN hyperlens slab, two factors improve the quality of the imaged hyperlens fields: (a) the higher launching

efficiency from metal object underneath $\mathrm{hBN}$, and (b) the lower losses of $\mathrm{h}^{11} \mathrm{BN}$. The launching efficiency is related to either the polarizability of the launcher (nickle disk here) ${ }^{23}$ or the momentum difference between $\mathrm{hBN} /$ launcher and suspended $\mathrm{hBN}^{24,25}$. As such, the launching efficiency either maintains a constant or slowly decreases with increasing frequnency (SI, section VIII). To describe the attenuation of the $\mathrm{HPhPs}$ as they propagate through the $\mathrm{hBN}$ slab, the quality factor $(\mathrm{Q})$ is defined as follows ${ }^{26,27}$ :

$$
Q=\frac{k^{\prime}}{k^{\prime \prime}}=4 \pi N
$$

where $k^{\prime}$ and $k^{\prime \prime}$ are the real and imaginary parts of the wavevector, respectively, and $N$ defines the number of cycles before the HPhP wave decays to $1 / e$. Thus, increasing $Q$ implies a stronger hyperlens signal, and a higher amplitude of the first and each subsequent hot ring observed within the hyperlens fields. To find 
$k^{\prime \prime}$, an analytical model ${ }^{3,25-27}$ is employed to calculate both the real and imaginary part of the HPhP wavevectors propagating in $\mathrm{hBN}$ over any material:

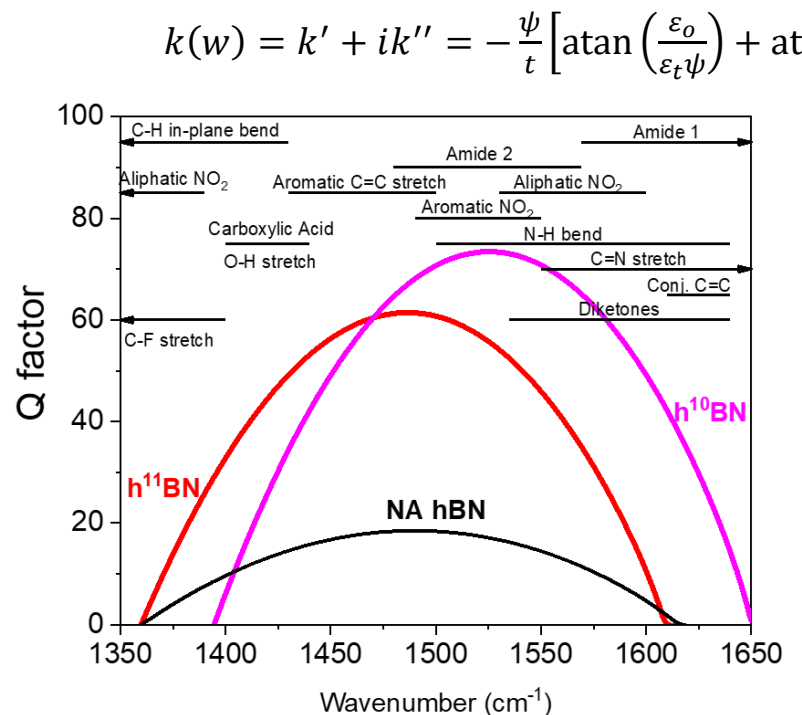

Figure 5: The $Q$ factor for hyperlens imaging for different boron isotope concentrations. The $Q$ factor for hyperlens imaging as defined in the main text is presented for $N A-h B N$ (black curve) along with both $h^{11} B N$ (red curve) and $h^{10} B N$ (magenta curve) materials. The spectral range associated with a number of different chemical vibrational modes of interest are also provided, each designated by the extent of the black lines, with arrows indicating the spectral range extends beyond that of the Reststrahlen band.

where $t$ represents the hBN thickness, $\varepsilon_{o}$ and $\varepsilon_{s}$ are the complex dielectric functions of air and the substrate, while $l$ represents the HPhP mode order. Using this analytical model, we plot the $Q$ of $\mathrm{h}^{11} \mathrm{BN}$ as a function of the incident (imaging) frequency in Fig. 5, and we find that the $Q$ is highest at around 1490 $\mathrm{cm}^{-1}$ for both NA and $\mathrm{h}^{11} \mathrm{BN}$. As such, we expect the best image quality for a given $\mathrm{hBN}$-based hyperlens to occur near this peak- $Q$ frequency, which agrees with our experimental observations (Fig. 4). Although the analytical solution is not quantitatively accurate when the HPhP wavevectors are not significantly larger than the free-space values (more discussions are in SI, section VIII), this approach still provides qualitative guidance towards optimizing hyperlens operation and design in this regime as well.

While the image quality is clearly influenced by the imaging frequency, the isotopic enrichment is still the dominant driver in the improved image quality we report. By comparing the $Q$ of $\mathrm{h}^{11} \mathrm{BN}$ and NA hBN, we estimate that $\mathrm{h}^{11} \mathrm{BN}$ should provide an approximate three-fold increase in transmission, causing better image quality over the entire URB, as shown in Fig. 5. Consistent with this, the PSNR of hyperlens fields collected using $\mathrm{h}^{11} \mathrm{BN}$ are indeed three times larger than those using NA hBN, as discussed earlier and presented in Fig. 2, Fig. S5, Table S1. 
It is important to note that spectral overlaps between the URB of $\mathrm{hBN}$ and the molecular fingerprint region of the MIR enables the potential for resonant hyperlens imaging (Fig. 5); that is, imaging of biologically relevant species at frequencies corresponding to the vibrational resonances of the materials for chemical identification. This could be especially beneficial for biological studies as the molecular vibrational bands of many molecules, e.g., the amide I and II bands of proteins ${ }^{28}$, fall within the frequency range of the URB of hBN. For resonant imaging of bio-materials, the largest hyperlens field contrast will occur at the chemical vibrational frequencies ${ }^{21,22}$, and $\mathrm{h}^{11} \mathrm{BN}$ and $\mathrm{h}^{10} \mathrm{BN}$ together cover a broad range of vibrational frequencies of interest, as shown by the overlayed black lines in Fig. 5. Thus, for different purposes, these two monoisotopic hBN materials (and potentially $\mathrm{h}^{10} \mathrm{~B}^{15} \mathrm{~N}$ and $\mathrm{h}^{11} \mathrm{~B}^{15} \mathrm{~N}$ if grown) could be used to optimize such imaging methodologies.

\section{Conclusion}

In conclusion, through the exploitation of ultra-low loss $\mathrm{h}^{11} \mathrm{BN}$ hyperlens devices, we demonstrate the ability to resolve the smallest features we fabricated $(\sim 44 \mathrm{~nm})$, showing a record-high imaging resolution of at least $\lambda_{F S} / 154$, which is a nearly four-fold improvement over previously reported data using NA hBN material ${ }^{5,6}$. Additionally, we demonstrate the ability to discern four closely spaced $\sim 100 \mathrm{~nm}$ disks, even at interparticle separations of $25 \mathrm{~nm}$, indicating the potential for imaging objects with separations that are on the order of $\lambda_{F S} / 270$. Note that the high PSNR of $h^{11} \mathrm{BN}$ hyperlens fields suggests that the ultimate resolution limit has yet to be reached. Furthermore, the transmission of $h^{11} \mathrm{BN}$ is predicted to be three times higher than NA hBN, which is consistent with the three-fold increasement of PSNR measured experimentally, providing significant opportunities in employing thicker and/or curved hyperlenses necessary for far-field hyperlens designs. Further, using a numerical algorithm, we demonstrate precise image reconstruction and accurately retrieve the details of the embedded $2 x 2, \sim 100 \mathrm{~nm}$ disk array with $\sim 25$ nm gaps, validating this as a potential methodology for detecting unknown sub-diffractional objects. Combining the numerical algorithm and ultra-low loss monoisotopic hBN, we provide the broad potential of imaging modalities, such as resonant hyperlens imaging with incident frequencies coincident with the 
vibrational frequencies of materials. We also offer analytical calculations of the frequency dependence of $Q$, necessary for realizing optimal performance. Therefore, this work also more broadly serves as a roadmap for designing hyperlenses using other newly discovered hyperbolic materials ${ }^{12,20,29-33}$ for sub-diffractional imaging, focusing and label-free detection.

Acknowledgement. Research on hBN nano-structures at Columbia is supported by AFOSR FA9550-151-0478. Imaging applications of isotopically pure hBN at Columbia are supported ONR-N000014-18-12722. The development of nano-imaging capabilities at Columbia is supported as part of Programmable Quantum Materials, an Energy Frontier Research Center funded by the U.S. Department of Energy (DOE), Office of Science, Basic Energy Sciences (BES), under award DE-SC0019443”. DNB is the Vannevar Bush Faculty Fellow ONR-VB: N00014-19-1-2630. HL device fabrication at NRL was supported from ASEE fellowship for GRSI through the Office of Naval Research distributed by the Naval Research Lab's Nanoscience Institute JDC. Computational imaging at Princeton is supported by the Air Force Office of Scientific Research (AFOSR) under Grant FA9550-18-1-029. Support for hBN crystal growth from the Office of Naval Research from award N00014-20-1-2427 is appreciated. Imaging work at Vanderbilt was supported for M.H. and J.D.C. by ONR-N00014-18-1-2107, while J.R.M. was supported by the National Science Foundation, Division of Materials Research under grant number 1904793.

\section{REFERENCES}

1 Poddubny, A., lorsh, I., Belov, P. \& Kivshar, Y. Hyperbolic metamaterials. Nature photonics 7, 948 (2013).

2 Caldwell, J. D. et al. Sub-diffractional volume-confined polaritons in the natural hyperbolic material hexagonal boron nitride. Nature communications 5, 5221 (2014).

3 Dai, S. et al. Tunable phonon polaritons in atomically thin van der Waals crystals of boron nitride. Science 343, 1125-1129 (2014).

4 Ishii, S., Kildishev, A. V., Narimanov, E., Shalaev, V. M. \& Drachev, V. P. Sub - wavelength interference pattern from volume plasmon polaritons in a hyperbolic medium. Laser \& Photonics Reviews 7, 265-271 (2013).

$5 \mathrm{Li}$, P. et al. Hyperbolic phonon-polaritons in boron nitride for near-field optical imaging and focusing. Nature communications 6, 7507 (2015).

6 Dai, S. et al. Subdiffractional focusing and guiding of polaritonic rays in a natural hyperbolic material. Nature Communications 6, 6963, doi:10.1038/ncomms7963 (2015).

7 Liu, Z., Lee, H., Xiong, Y., Sun, C. \& Zhang, X. Far-field optical hyperlens magnifying sub-diffractionlimited objects. science 315, 1686-1686 (2007).

8 Rho, J. et al. Spherical hyperlens for two-dimensional sub-diffractional imaging at visible frequencies. Nature communications 1, 1-5 (2010).

9 Lu, D. \& Liu, Z. Hyperlenses and metalenses for far-field super-resolution imaging. Nature communications 3, 1205 (2012). 
Ma, C. \& Liu, Z. A super resolution metalens with phase compensation mechanism. Applied Physics Letters 96, 183103 (2010).

11 Dai, S. et al. Internal nanostructure diagnosis with hyperbolic phonon polaritons in hexagonal boron nitride. Nano letters 18, 5205-5210 (2018).

12 Sun, J., Litchinitser, N. M. \& Zhou, J. Indefinite by nature: from ultraviolet to terahertz. Acs Photonics 1, 293-303 (2014).

13 Hoffman, T. B., Clubine, B., Zhang, Y., Snow, K. \& Edgar, J. H. Optimization of Ni-Cr flux growth for hexagonal boron nitride single crystals. Journal of crystal growth 393, 114-118 (2014).

14 Liu, S. et al. Single crystal growth of millimeter-sized monoisotopic hexagonal boron nitride. Chemistry of Materials 30, 6222-6225 (2018).

Liu, S. et al. Large-scale growth of high-quality hexagonal boron nitride crystals at atmospheric pressure from an Fe-Cr flux. Crystal growth \& design 17, 4932-4935 (2017). 134 (2018).

Cuscó, R., Edgar, J. H., Liu, S., Li, J. \& Artús, L. Isotopic Disorder: The Prevailing Mechanism in Limiting the Phonon Lifetime in Hexagonal BN. Physical Review Letters 124, 167402 (2020). Gerchberg, R. W. A practical algorithm for the determination of phase from image and diffraction plane pictures. Optik 35, 237-246 (1972). Barsi, C., Wan, W. \& Fleischer, J. W. Imaging through nonlinear media using digital holography. Nature Photonics 3, 211-215 (2009). Álvarez - Pérez, G. et al. Infrared Permittivity of the Biaxial van der Waals Semiconductor a MoO3 from Near - and Far - Field Correlative Studies. Advanced Materials, 1908176 (2020). Wang, C.-F., Kafle, B., Tesema, T. E., Kookhaee, H. \& Habteyes, T. G. Molecular Sensitivity of NearField Vibrational Infrared Imaging. The Journal of Physical Chemistry C (2020).

Bylinkin, A. et al. Real-space observation of vibrational strong coupling between propagating phonon polaritons and organic molecules. Nature Photonics, 1-6 (2020).

23 Dai, S. et al. Efficiency of launching highly confined polaritons by infrared light incident on a hyperbolic material. Nano letters 17, 5285-5290 (2017).

24 de Galarreta, C. R. et al. Nonvolatile Reconfigurable Phase - Change Metadevices for Beam Steering in the Near Infrared. Advanced Functional Materials 28, 1704993 (2018).

$\mathrm{He}, \mathrm{M}$. et al. Guided Mid-IR and Near-IR Light within a Hybrid Hyperbolic-Material/Silicon Waveguide Heterostructure. arXiv preprint arXiv:2006.06920 (2020).

26 Fali, A. et al. Refractive Index-Based Control of Hyperbolic Phonon-Polariton Propagation. Nano letters 19, 7725-7734 (2019).

27 Dai, S. et al. Hyperbolic phonon polaritons in suspended hexagonal boron nitride. Nano letters 19, 1009-1014 (2018).

28 Chatzakis, l. et al. Strong confinement of optical fields using localized surface phonon polaritons in cubic boron nitride. Optics letters 43, 2177-2180 (2018).

29 Korzeb, K., Gajc, M. \& Pawlak, D. A. Compendium of natural hyperbolic materials. Optics express 23, 25406-25424 (2015).

30 Low, T. et al. Polaritons in layered two-dimensional materials. Nature materials 16, 182-194 (2017).

31 Ma, W. et al. In-plane anisotropic and ultra-low-loss polaritons in a natural van der Waals crystal. Nature 562, 557 (2018).

32 Zheng, Z. et al. Highly confined and tunable hyperbolic phonon polaritons in van der Waals semiconducting transition metal oxides. Advanced Materials 30, 1705318 (2018).

33 Taboada-Gutiérrez, J. et al. Broad spectral tuning of ultra-low-loss polaritons in a van der Waals crystal by intercalation. Nature materials, 1-5 (2020). 
34 Vuong, T. et al. Isotope engineering of van der Waals interactions in hexagonal boron nitride. Nature materials 17, 152 (2018).

35 Kretinin, A. et al. Electronic properties of graphene encapsulated with different two-dimensional atomic crystals. Nano letters 14, 3270-3276 (2014).

36 Chen, X. et al. Modern Scattering - Type Scanning Near - Field Optical Microscopy for Advanced Material Research. Advanced Materials, 1804774 (2019).

\section{METHODS}

Sub-diffractional Object Fabrication: To realize the hyperlens concept, sub-diffractional nickel (Ni) disks were patterned on $\mathrm{SiO}_{2}$ substrates using the Raith-150 electron beam writer. For the fabrication of the fine features by e-beam lithography, we used the ZEP520 resist. The e-write was followed by resist development, metal deposition and lift-off to realize the 30-nm thick Ni structures.

Device fabrication: We used two forms of hBN crystals in our study, the naturally abundant and ${ }^{11} \mathrm{~B}$ enriched. The NA was procured from HQ Graphene, while the monoisotopic hBN crystals were grown by the precipitation $\mathrm{hBN}$ synthesized from nitrogen and elemental ${ }^{11} \mathrm{~B}(99.4 \%)$ from a molten metal flux of nickel and chromium. The crystal growth techniques utilized for $\mathrm{h}^{11} \mathrm{BN}$ are described in reference ${ }^{14,34}$. Both types of hBN flakes of various thickness were obtained by standard exfoliation from the bulk crystals onto a PMMA/PVA (Poly-vinyl acetate) bi-layer stack on a silicon wafer. The PMMA/hBN stack is carefully and precisely transferred onto the fabricated hyperlens pattern, finally washing off the PMMA to obtain the hBN-based hyperlens devices. More details of this transfer technique are given in $\operatorname{Ref}^{35}$.

Near-field imaging (Infrared s-SNOM Measurements and Analysis): Infrared nano-imaging was performed with a commercial (Neaspec $\mathrm{GmbH}$ ) scattering-type scanning near-field optical microscope (sSNOM) based on a tapping-mode atomic force microscope. A quantum cascade laser (DRS Daylight Solutions) was used as the light source, which is focused onto a metallic tip oscillating at a tapping frequency of $\sim 250 \mathrm{kHz}$ with a tapping amplitude of around $60 \mathrm{~nm}$. The scattered light was detected using a liquid nitrogen cooled $\mathrm{HgCdTe}(\mathrm{MCT})$ detector. To suppress far-field background signals, the detected signal was demodulated at a harmonic $n$ of the tapping frequency. In this work, we used $n=3$. Additional 
description of the s-SNOM technique is available elsewhere ${ }^{36}$. The data presented in the paper was collected from two systems: system 1 at Columbia Univerisity, and system 2 at Vanderbilt University.

Numerical Simulation of Near fields and spectra: Electromagnetic simulations of hyperlense fields on the top of hBN were carried out using the frequency-domain solver in CST Studio Suite 2018. The model consisted of a 125-nm thick slab of hBN (lateral size $5 \mathrm{um}$ ) on top of Ni disks matching the experiment, which were placed onto a 270-nm thick thermal $\mathrm{SiO}_{2}$ substrate. This model was excited through the substrate with normal incidence plane waves using Floquet boundary conditions. In order to minimize speckle in the simulated fields, the nickel disk edges were rounded (blend radius 10-nm), and the mesh density was enhanced to a maximum tetrahedron dimension of 3-nm within the nickel disks themselves. Furthermore, the mesh density was also enhanced to $30 \mathrm{~nm}$ within the $\mathrm{hBN}$ in a $1 \mathrm{um}$ square above the disks. Further, for the simulations in Fig. 3, the damping constant of the $\mathrm{h}^{11} \mathrm{BN}$ was increased three-fold. We rotated the extracted field of one polarization and summed it with the unrotated one to reproduce the experimentally measured image.

Data Availability: The data that supports the finding of this study are available from the corresponding author on reasonable request. 


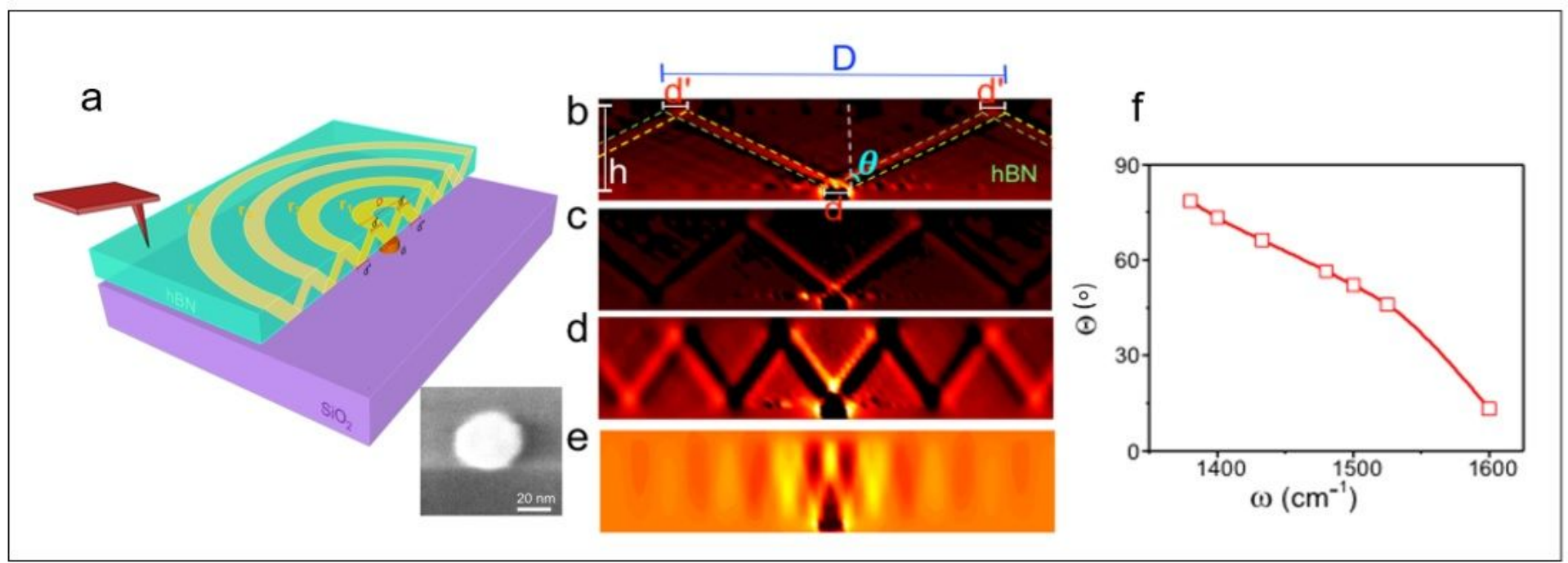

\section{Figure 1}

Sub-diffractional focusing and imaging through $\mathrm{hBN}$ a Schematic illustration of the hot rings/fringes on the top surface and within the hBN as a result of multiple reflection of the HPhPs launched from the edges of the sub-diffractional nickel disks embedded beneath 125-nm thick h11BN slab. Inset: a SEM image of the 44-nm disk. b-e The simulated cross-sectional E-field distribution for a 40-nm disk embedded under a 125-nm thick h11BN flake at $\omega=1433 \mathrm{~cm}-1,1500 \mathrm{~cm}-1,1560 \mathrm{~cm}-1,1600 \mathrm{~cm}-1$ respectively. $\mathrm{f}$, The calculated propagation angle $(\theta)$ of HPhPs within h11BN as a function of frequency. Open squares correspond to spectral positions for experiments and simulations discussed. 

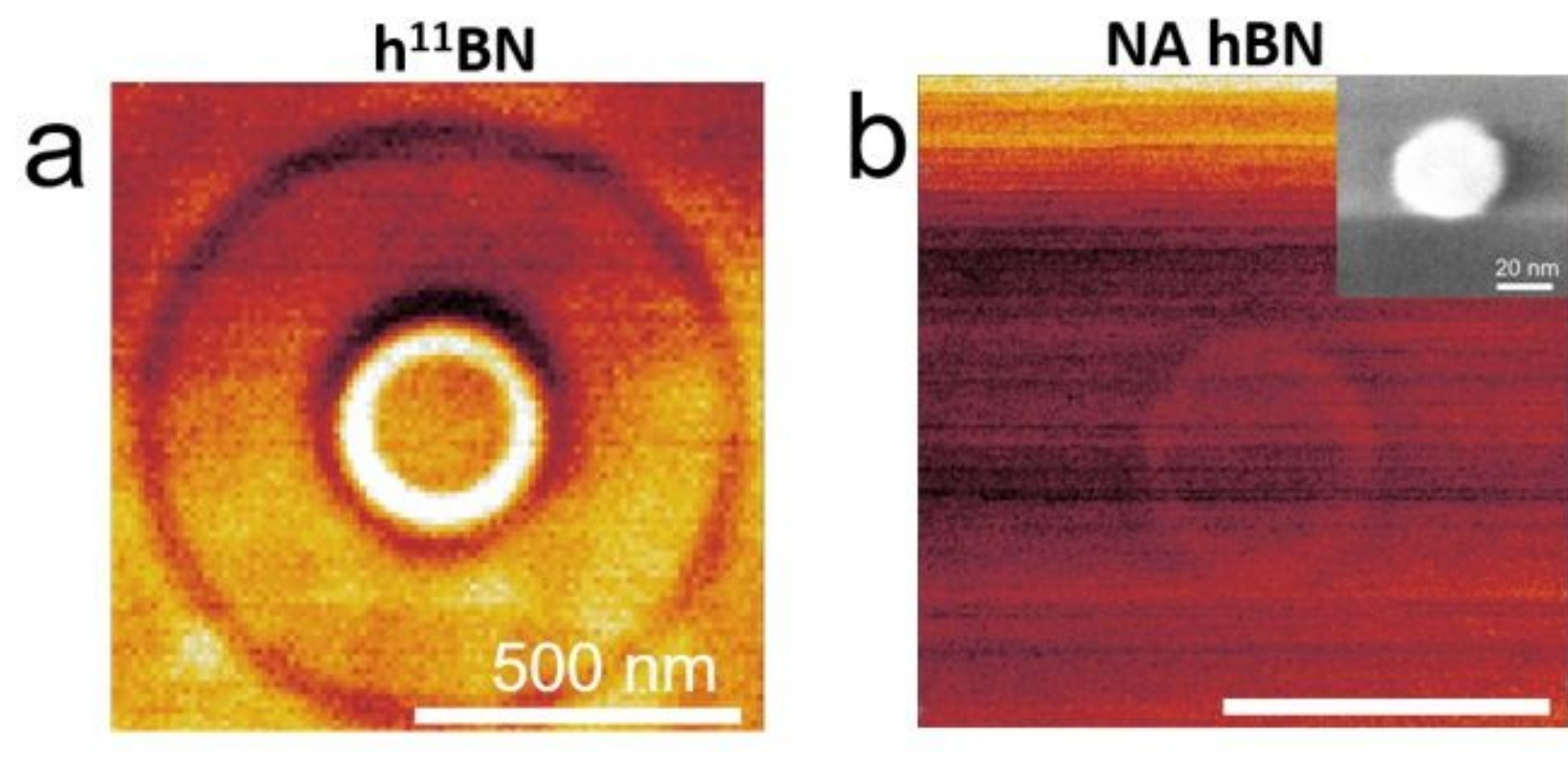

\section{$\operatorname{Max}$}
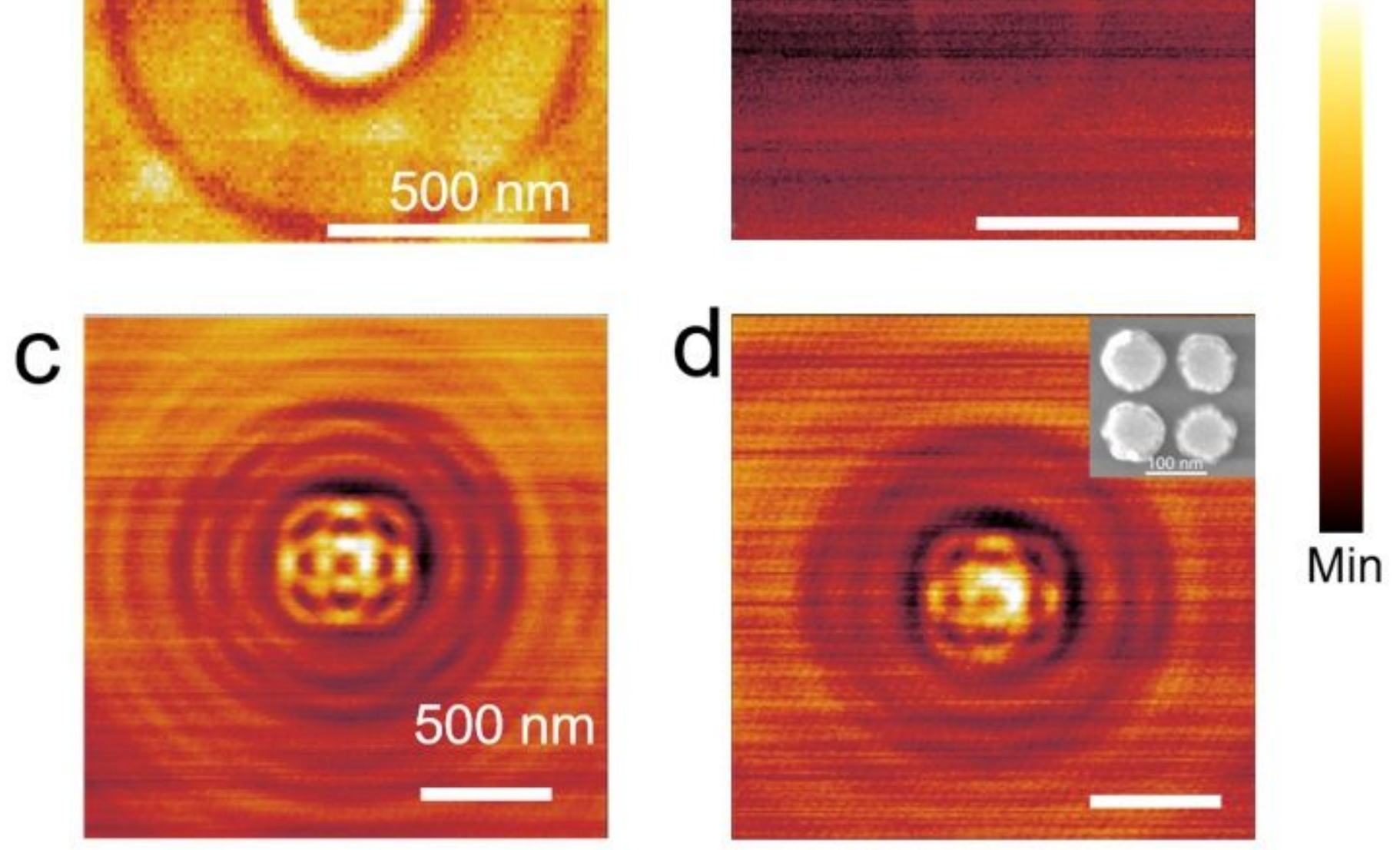

Figure 2

Experimental demonstration of super high-resolution sub-diffractional focusing and imaging through natural and monoisotopically enriched $h B N$. $(a, b)$. Near field amplitude image of $44 \mathrm{~nm}$ square at $\omega=1500 \mathrm{~cm}-1$ for 11B and NA-hBN. (c, d) Near field amplitude image of a 2x2, $100 \mathrm{~nm}$ disk array with 25 $\mathrm{nm}$ gap separation at $\omega=1480 \mathrm{~cm}-1$ for $11 \mathrm{~B}$ and NA-hBN. Corresponding SEM images are inseted. 
Back-propagation: $\quad \varepsilon_{\text {prop }}^{(n)}=\mathcal{P}_{-d}\left[E_{\text {prop }}^{(n)}\right]$

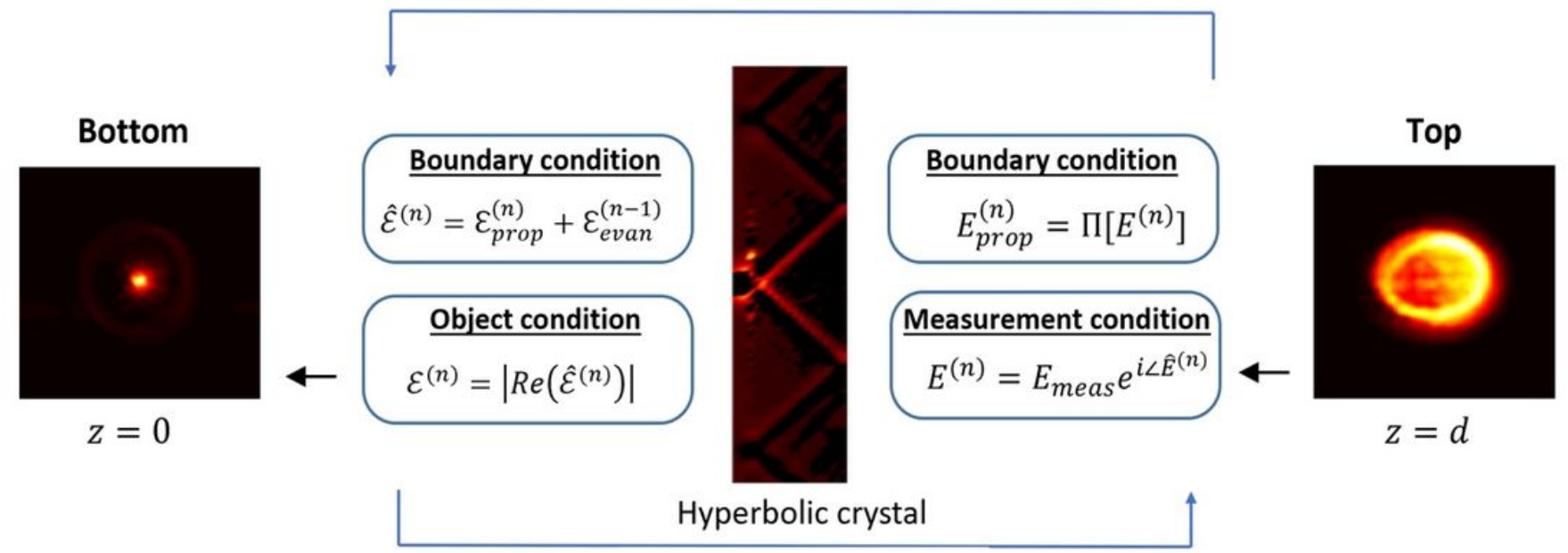

Forward propagation: $\quad \hat{E}^{(n)}=\mathcal{P}_{d}\left[\mathcal{E}^{(n)}\right]$

\section{Figure 3}

Algorithm flowchart. The algorithm iterates between the experimentally measured field amplitude at the top of the crystal and the object field at the bottom of the crystal. The right-most image shows a sample measurement of the crystal output, the central image depicts approximate ray paths within the crystal, and the leftmost image represents the input field reconstruction after 200 iterations. We start the algorithm by enforcing the measurement condition; is the measured field, denotes the phase of the complex field (to be found), and represents the iteration number. The projection operator extracts the propagating modes from by enforcing the boundary constraints on the input and output faces of the crystal. The angular-spectrum propagation operator transports the propagating modes of the input field by a distance. To match the boundary conditions at the bottom of the crystal, we add the evanescent modes (obtained from the previous iteration ) to get the total field. We further apply the small object constraint to get our reconstruction. Subscripts and represent propagating and evanescent components of a given electric field, respectively, represents the real part of , and represents an absolute value operation. 

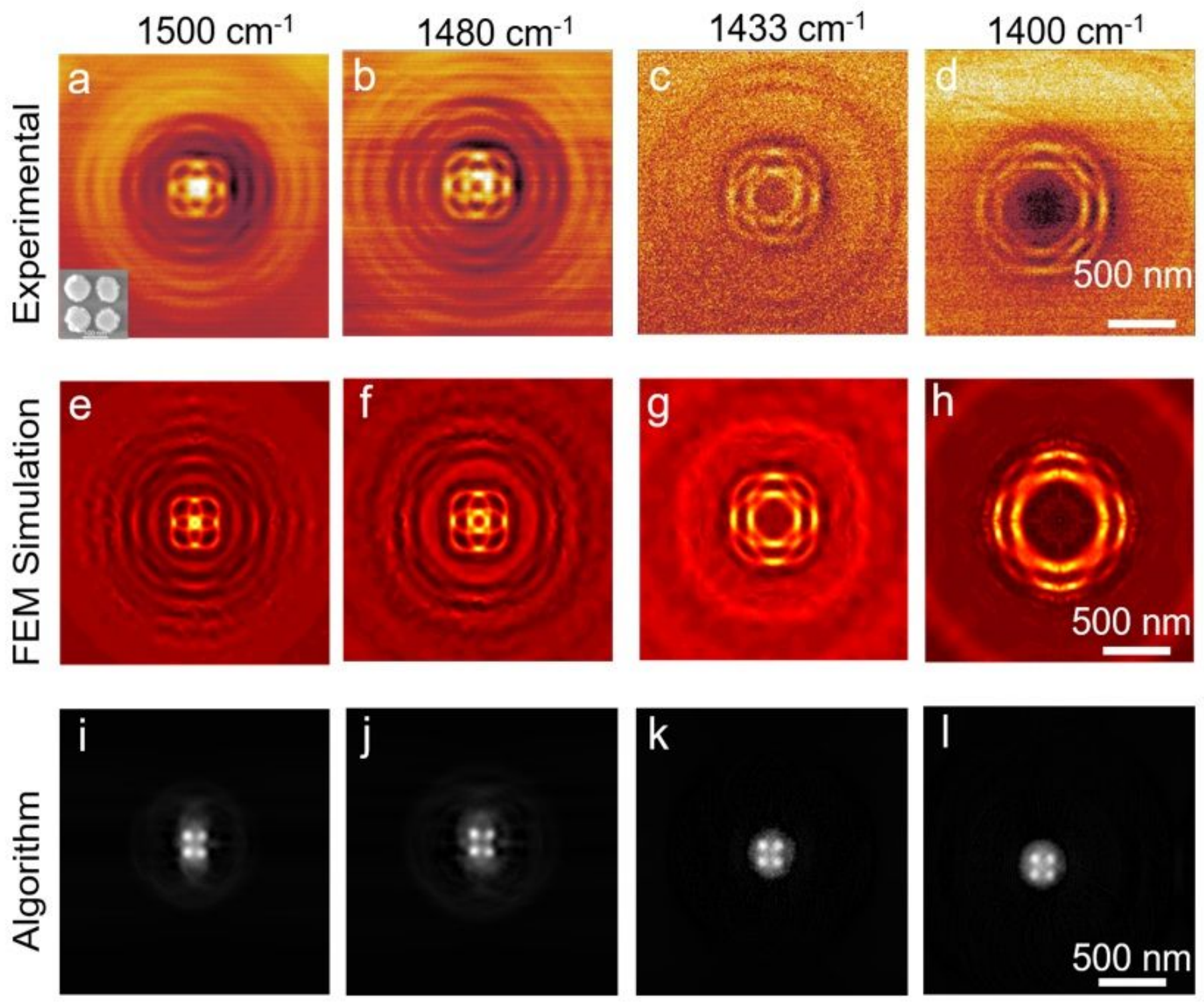

Figure 4

Frequency-dependent hyperlens fields and reconstruction. (a-d) Near-field amplitude images of $\sim 100-\mathrm{nm}$ disk array with 25-nm inter-particle gaps, showing a strong dependence on the HPhP propagation angle. (e-h) Corresponding FEM simulations. (i-I) Reconstructed underlying structures from the corresponding sSNOM images. 


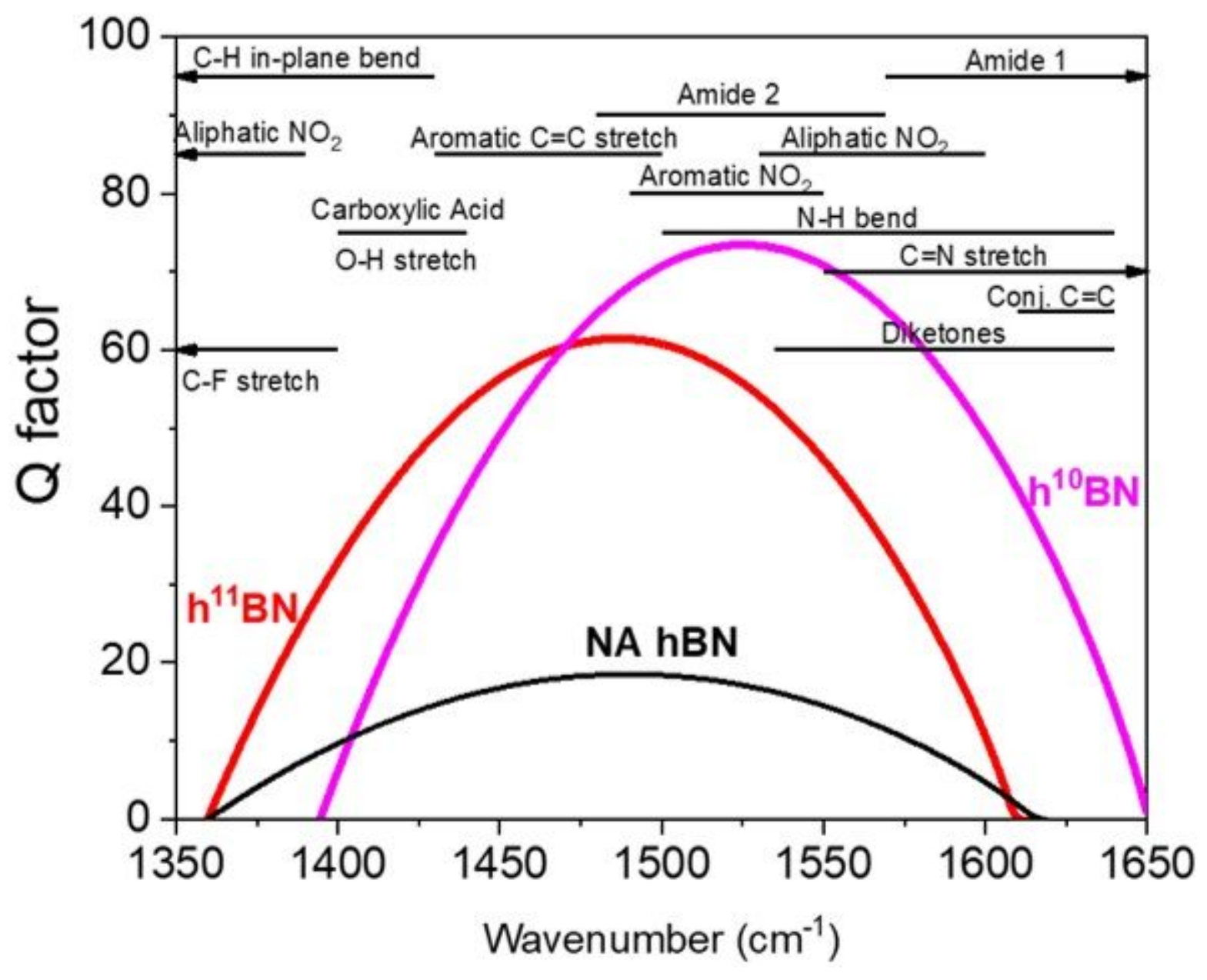

Figure 5

The $\mathrm{Q}$ factor for hyperlens imaging for different boron isotope concentrations. The $\mathrm{Q}$ factor for hyperlens imaging as defined in the main text is presented for NA-hBN (black curve) along with both h11BN (red curve) and h10BN (magenta curve) materials. The spectral range associated with a number of different chemical vibrational modes of interest are also provided, each designated by the extent of the black lines, with arrows indicating the spectral range extends beyond that of the Reststrahlen band.

\section{Supplementary Files}

This is a list of supplementary files associated with this preprint. Click to download.

- HLpaperSlsubmissionFeb2021.docx 